\title{
GNAS1 mutation analysis in gastrointestinal tumors
}

\author{
Bernhard Maria Walther, Ina Walther, Yuan Chen, Iver Petersen \\ Institute of Pathology, University Hospital Jena, Friedrich-Schiller-University, Jena, Germany
}

\begin{abstract}
GNAS1 codes for a part of the $\alpha$-stimulatory subunit (Gs $\alpha$ ) of the G protein. Mutation of GNAS1 has been frequently found in myxoid soft tissues, however, in gastrointestinal tumors, little is known about the mutation status of GNAS1. The aim of the study was to analyze the occurrence of GNAS1 mutations in different gastrointestinal, gastroenteropancreatic neuroendocrine tumors (GEP-NETs) and colorectal tumors. Mutation status of GNAS1 exon 8 was analyzed in one hundred thirty-five formalin-fixed, paraffin-embedded (FFPE) gastrointestinal tumor samples including 45 tubular-villous adenomas, 11 tubular adenomas, 6 villous adenomas, 10 hyperplastic gastric polyps, 31 GEP-NETs and 32 colorectal adenocarcinomas by using polymerase chain reaction (PCR) and direct sequencing. Five GNAS1 mutations were found in 2 tubular-villous adenomas, 2 villous adenomas and 1 colorectal adenocarcinoma. No mutations were detected in the tubular adenomas, the hyperplastic gastric polyps or GEP-NETs. GNAS1 mutation is not a frequent molecular event in GEP-NETs or hyperplastic gastric polyps. The study confirms the presence of GNAS1 mutations in colon tumors with villous differentiation. (Folia Histochemica et Cytobiologica 2014, Vol. 52, No. 2, 90-95)
\end{abstract}

Key words: GNAS1 mutations; gastric cancer; intestinal tumors; CRC; GEP-NETs

\section{Introduction}

Cancer is worldwide and especially in more industrialized nations a major reason for hospitalization and treatment. In 2008, there were 447000 new cases of colorectal cancer in the European Union [1]. About $65 \%$ of all new cases occur in high-income countries. Behavioral risk factors including diet, alcohol intake and physical inactivity account for a large proportion of cases. In Europe, colorectal cancer (CRC) is the second most common cause of cancer death in both men and women. It is the second most common form of cancer in women, after breast cancer, and the third most common in men, after lung and prostate cancer [1]. Although the incidence of gastric cancer is decreasing, there were still 159900 new cases in Europe in 2006, and about 118200 deaths, representing the fifth highest incidence and fourth highest cause of cancer-related death [2]. To achieve a reduced mortality of gastrointestinal cancer, a better under-

Correspondence address: I. Petersen, M.D. Institute of Pathology, University Hospital, Friedrich-Schiller-University, Jena

Ziegelmühlenweg St. 1, 07743 Jena, Germany tel.: +49 (0)3641 933120, fax: +49 (0)3641933111

e-mail: iver.petersen@med.uni-jena.de standing of the molecular mechanisms underlying carcinogenesis is essential which eventually will lead to early diagnosis of the disease.

The GNAS1 gene is located on chromosome 20q13.3. It has 13 exons and can undergo alternative splicing [3, 4]. GNAS1 codes for the $\alpha$-stimulatory subunit $(\mathrm{Gs} \alpha)$ of heterotrimeric G proteins. Gs $\alpha$ activates the adenylyl cyclase which then catalyzes cyclic adenosine monophosphate (cAMP) production and can thereby regulate downstream signaling pathways [6]. As a vital parameter in cellular signaling Gs $\alpha$ is ubiquitously found [7]. Different diseases are described with gain-of-function and loss-of-function mutations such as McCune-Albright-Syndrome, a disease which combines café-au-lait spots, polyostotic fibrous dysplasia and endocrinopathies [8], fibrous dysplasia itself [9] or endocrinological diseases like pseudohypoparathyroidism [10] with various mutation hotspots [11]. How imprinting mechanism of this gene leads to different diseases partly depending on parent's gender, is not totally understood [5]. Because of the widespread occurrence of G-protein-coupled receptors mutations the gene could in principle be involved in a large number of different tumor types. And indeed, it was shown that GNAS1 mutation could be found in different neoplasia throughout the whole body ranging from pituitary gland tumors [12] over 
Table 1. Study collective and experimental results

\begin{tabular}{|l|c|c|c|c|}
\hline Entity & Number of samples & Age & Mutations found & Kind of mutation \\
\hline Tubular-villous adenoma & 45 & \multirow{2}{*}{ Mean 76; range 47-85 } & 2 & R 201 H \\
\cline { 1 - 2 } \cline { 5 - 5 } Tubular adenoma & 11 & & No & \\
\cline { 1 - 2 } Villous adenoma & 6 & Mean 72; range 49-85 & No & \\
\hline Hyperplastic gastric polyps & 10 & Mean 56; range 19-87 & No & \\
\hline GEP-NET's & 31 & Mean 77; range 58-85 & 1 & R 201 C \\
\hline Colorectal adenocarcinoma & 32 & & $\mathbf{5}$ & \\
\hline All & $\mathbf{1 3 5}$ & & \\
\hline
\end{tabular}

pancreatic intraductal papillary mucinous neoplasm (IPMN) [13, 14], low-grade appendiceal mucinous neoplasia [15], villous adenomas of the colorectum [16] to colorectal carcinoma [17]. However, the mutation status of GNAS1 in gastrointestinal cancer has not yet been well investigated. So far only a handful of publications addressed this issue. Therefore in our study we focused on mutation analysis of GNAS1 in different neoplasia of the digestive tract.

\section{Material and methods}

Tumor samples. Altogether 135 gastrointestinal tumor samples from different entities were included in our study (Table 1). Among them, 45 were tubular-villous adenomas, 11 tubular adenomas, both of them ranging from low to high grade, 6 villous adenomas, a rare tumor type, 10 hyperplastic gastric polyps, 31 gastroenteropancreatic neuroendocrine tumors (GEP-NETs) and 32 colorectal adenocarcinomas. The tubular-villous adenomas were collected throughout the whole gastrointestinal tract and were found in following regions: sigmoid colon -13 , colon not otherwise specified -11 , descending colon -6 , cecum -5 , rectum -4 , transverse colon -3 , and ascending colon -3 . All entities described above were removed endoscopically or surgically, and were fixed in formalin and embedded in paraffin (FFPE). The GEP-NET collective represented a subgroup from a recently published series [18]. The grading for the tumor samples was defined according to the WHO classification of tumors of the digestive system [19]. The GEP-NETs grading was performed according to Capella et. al [20]. All samples were retrieved from the collection of the Institute of Pathology of the Jena University Hospital; the GEP-NETs from 1995 to 2008 and all the other samples from 2010 to 2012.

Genomic DNA isolation, PCR product purification. Genomic DNA was isolated from the 135 FFPE tumor samples by using a QiAmp ${ }^{\circledR}$ DNA Mini Kit (Qiagen, Hilden, Germany) following the guidelines of the manufacturer.
The primer pair: 5'-CTCTTTCCAAACTACTCCAGACC-3' (sense) and 5'-AGCTGGTTATTCCAGAGGGACT-3' (antisense) was used for the PCR amplification of GNAS1. PeqSTAR 96 Universal Gradient PCR thermocycler has been used for PCR amplification and PeqGold Hot Taq DNA polymerase (Peqlab Biotechnology GmbH, Erlangen, Germany) was applied. PCR was performed in a $50 \mu$ l volume (50-100 ng genomic DNA, $2.0 \mathrm{mM}$ dNTPs and $10 \mathrm{pmol}$ of primer pair) under the following conditions: $94^{\circ} \mathrm{C}$ $1 \mathrm{~min}, 56^{\circ} \mathrm{C} 45 \mathrm{~s}, 72^{\circ} \mathrm{C} 45 \mathrm{~s}$, for 40 cycles after an initial denaturation at $95^{\circ} \mathrm{C}$ for $15 \mathrm{~min}$ and a final elongation at $72^{\circ} \mathrm{C}$ for $7 \mathrm{~min}$. In each PCR run, positive and negative controls were included. The expected size of the GNAS1 product is 252-bp. All PCR products were visualized on a $1.5 \%$ agarose. PCR products were purified by using a DNA Clean\&Concentrator $^{\mathrm{TM}}$ 5-KIT (Zymo Research Europe, Freiburg Germany) according to the manufacturer's instructions. One hundred nanogram (100 ng) of purified PCR products were applied for direct sequencing by capillary electrophoresis (LGC Genomics, Berlin, Germany).

\section{Results}

Of all 135 samples examined, we found that only 5 cases $(3.7 \%)$ showed GNAS1 mutations (Table 1). In adenomas, 4 out of $62(6.45 \%)$ were mutated. One of the mutated adenoma could not be assigned to a specific region of the colon, whereas all other 3 were found in the rectum. The mutation pattern of all GNAS1 positive adenomas was a change from Arginine to Histidine (R201H; CGT > CAT) (Figure 1). Two of them were diagnosed as tubular-villous low grade adenomas and 2 were villous high grade adenomas, one with severe and one with moderate dysplasia. Out of the 32 colorectal carcinomas, only one positive case $(3.13 \%)$ was identified. It consisted of the recurrence of a mucinous rectal adenocarcinoma (rpT3, rpN0 $(0 / 5), \mathrm{L} 0, \mathrm{~V} 0, \mathrm{Pn} 0, \mathrm{G} 3, \mathrm{R} 0)$. In contrast to the mutations of adenomas, the nucleotide exchange resulted in the substitution of Arginine to Cysteine (R201C; 


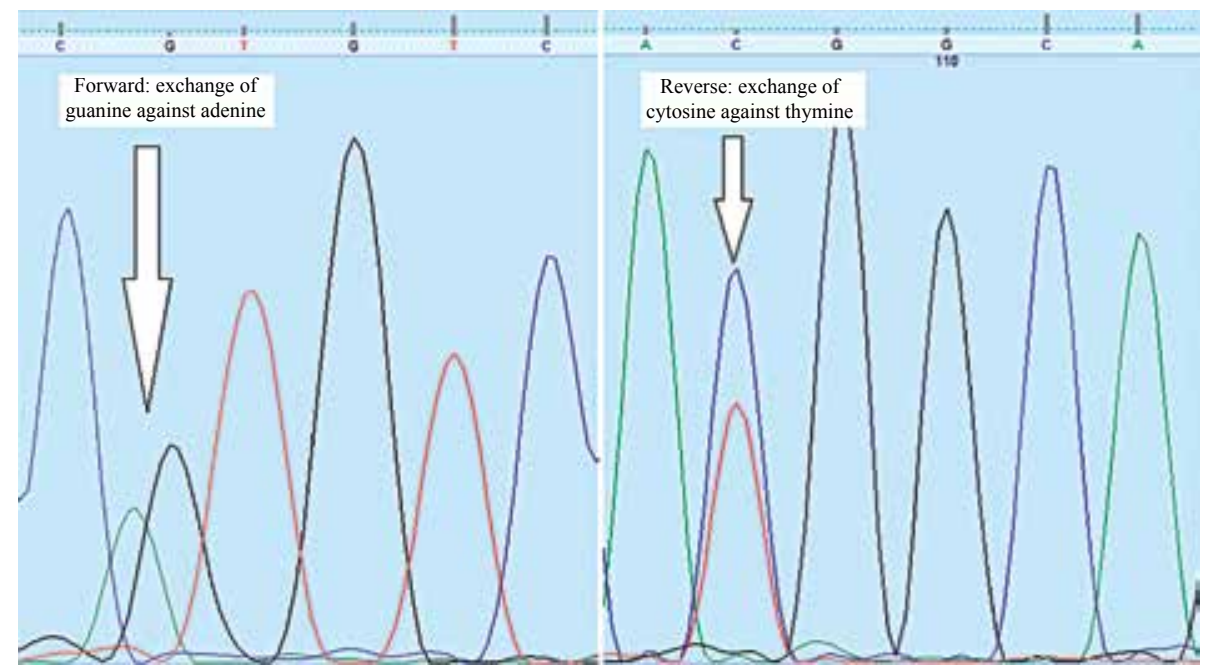

Figure 1. Sequence analysis of GNAS1 R201H mutation in a villous adenoma
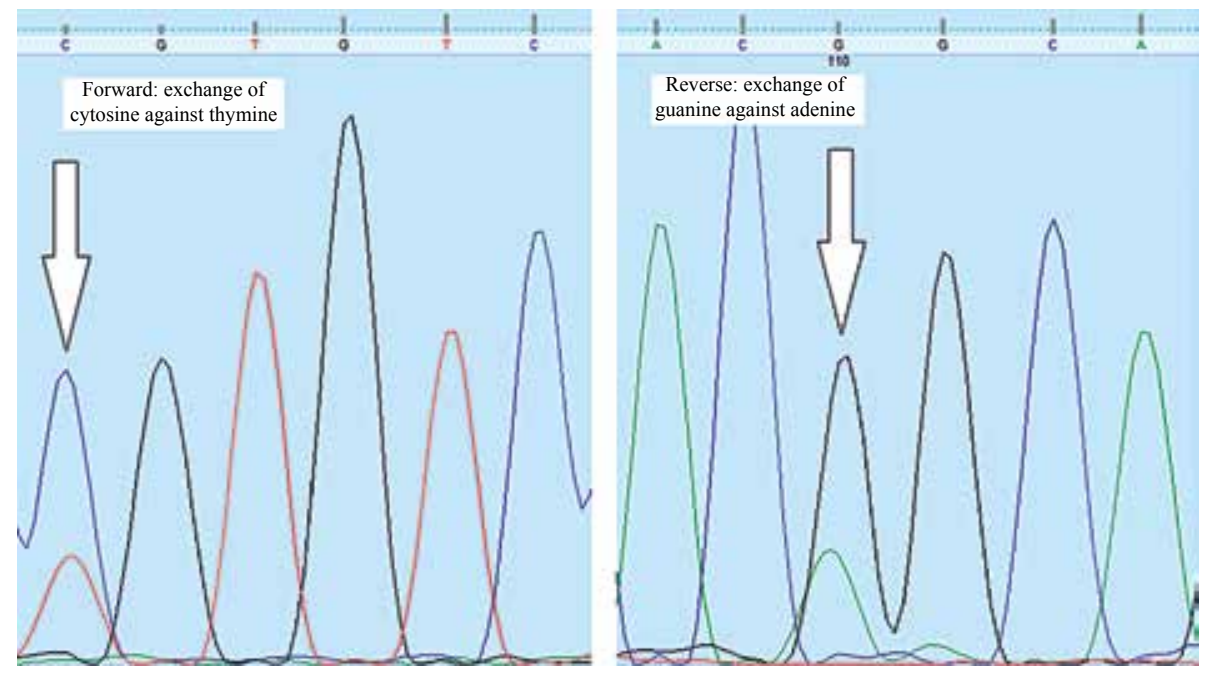

Figure 2. Sequence analysis of GNAS1 R201C of the colorectal carcinoma

CGT > TGT) (Figure 2). The carcinoma harbored an additional KRAS mutation (G13D; GGC > GAC). The histological analysis of the GNAS1-mutated tumors revealed no peculiar patterns compared to the non-mutated cases (Figure 3).

No GNAS1 mutation was found in 10 hyperplastic gastric polyps and 31 GEP-NETs.

\section{Discussion}

Many signaling pathways are connected with stimulatory $\mathrm{G}$ proteins. In particular, $\mathrm{G}$ protein-coupled receptors are the most common receptors which exert their function through nucleotide exchange on heterotrimeric $\mathrm{G}$ proteins [21]. These receptors undergo an exchange of GTP for GDP on the Gs $\alpha$-subunit initiating downstream signaling [22]. Mutations in Gs $\alpha$ itself, but also mutations in the binding pocket of $\mathrm{G}$ protein-coupled receptors, can affect their downstream function [23]. Being embedded with its seven transmembrane domains [24] in the double lipid layer of a cell, a $\mathrm{G}$ protein-coupled receptor transfers information from outside to the inside by the heterotrimeric $G$ protein and in particular by its GTP -bound alpha subunit. GNAS1 therefore represents a fundamental link to stimulate the cell in order to carry out its proper function. False stimulation or missing input leaves the cell without vital information or may initiate autonomous growth. Generally, mutation can cause an uninhibited activation [25] or a loss of func- 

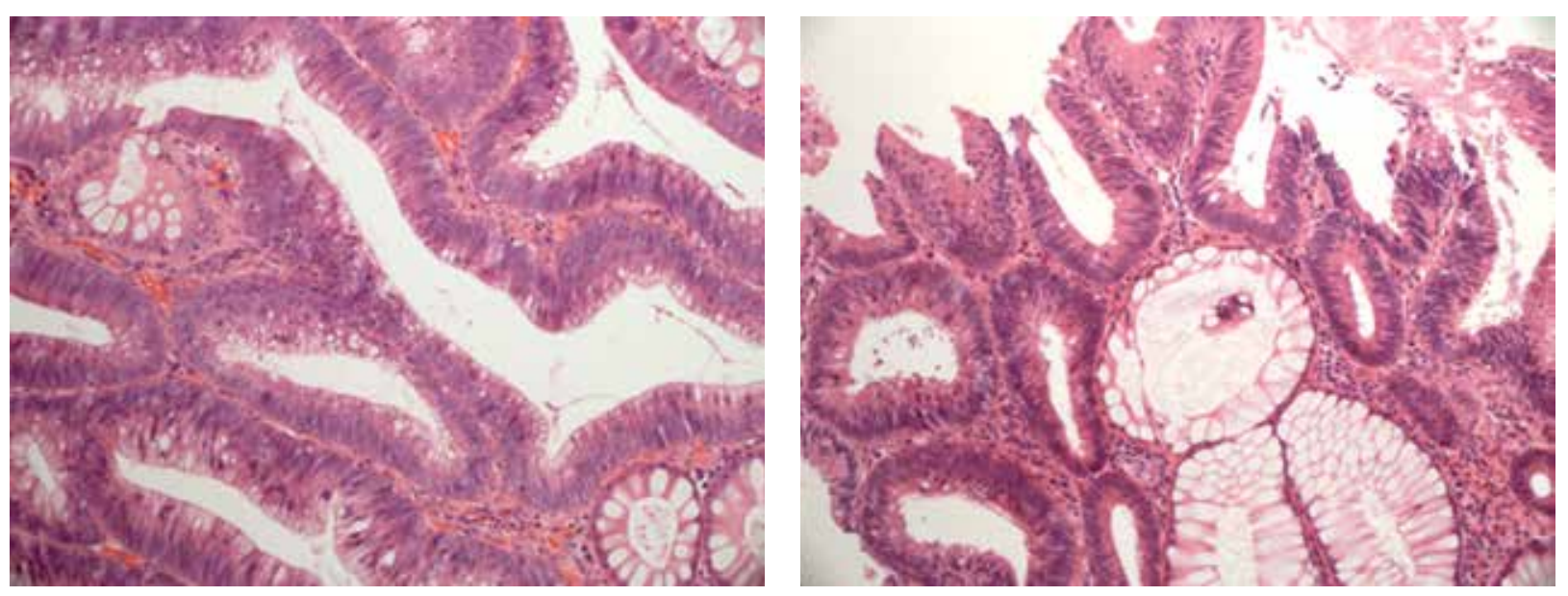

Figure 3. Representative histological images of two adenomas with GNAS R201H mutations in routine hematoxylin-eosin staining $(\times 200$ magnification $)$

tion [5]. Tissues with high cell turnover like epidermis or epithelium of gastrointestinal tract are especially vulnerable for acquiring mutations. It was proved long ago that RAS, a small G protein, is frequently mutated in colorectal cancer. GNAS1, however, was only recently identified to be mutated in a subset of colon neoplasia, i.e. villous adenoma [16]. Therefore, we decided to assess the most frequent mutation of GNAS1 gene by analyzing the FFPE samples of different subsets of gastrointestinal tumors.

In our study, among 45 tubular-villous adenomas, 2 cases showed GNAS1 mutations (4.44\%), which is in good agreement with the data from Yamada et al., who tested 35 tubular-villous adenomas and found only in one case (3\%) a GNAS1 mutation [16]. It indicates the possibility that GNAS1 mutations could be a factor in adenoma development. GNAS1 R201H and R201C are the most frequently missense mutations found in different non-cancer and cancer diseases $[9,26]$. Interestingly Yamada et al. found that 20 out of $24(83 \%)$ villous adenomas presented GNAS1 mutations [16]. We could prove two GNAS1 mutation out of 6 villous adenomas $(33.33 \%)$. This discrepancy between findings may be explained by a different racial background between Japanese and Caucasian populations. In fact, a very recent Japanese study of 12 villous adenomas in association with colon adenocarcinoma confirmed the high incidence since GNAS1 mutations were detected in 9 cases (75\%). Interestingly, the GNAS1 mutations were shared in only 3 of the 12 adenocarcinomas $(25 \%)$ of which 11 also harbored KRAS mutations [27]. It should be mentioned, however, that this study came from the same group that initially reported GNAS1 mutation in villous adenoma. Unfortunately, to our knowledge there are no independent reports from other groups confirming the high incidence of GNAS1 mutations in villous adenoma. Still, the data implicates that there may be a connection between a villous differentiation and the GNAS1 mutation in codon 201. The fact that also tubular-villous adenomas were mutated, suggests that even a small villous component raises the possibility of carrying a GNAS1 mutation. In contrast pure tubular adenomas showed no mutation, which corroborates the up to now published data [16]. Thus, it seems that there is no or only a very small relationship between tubular differentiation in colorectal adenoma and the GNAS1 mutation. This should be evaluated in further studies, with discrimination between the villous and tubular components and separate testing of the GNAS1 locus.

We found only one GNAS1 mutation in 32 tested colorectal adenocarcinomas. This is in concordance with the data from other groups regarding GNAS1 analysis. I.e., Yamada et al. found 2 mutations in 76 (3\%) colorectal adenocarcinomas [16]. Because of the above mentioned possible connection between villous differentiation in adenoma and the GNAS1 mutation, we wanted to examine if there was a similar villous component in our GNAS1 mutation positive tested CRC. However, our assumption could not be confirmed, because no remarkable villous differentiations were found in this specific GNAS1 positive CRC. Furthermore, there was no noticeable distinction in the histological appearance between the one mutated sample and the other 31 colorectal adenocarcinomas. Further studies of CRC focusing on the histological appearance and their possible GNAS1 mutation should be performed to classify the importance of this mutation in the development of colorectal adenocarcinoma. The recent study by Sekine et al. might indicate that the mutation is relevant for tumor initiation but dispensable for malignant 
tumor progression. It confirms our observation of the potential coincidence with a KRAS mutation [27]. Another recent study of 428 colon adenocarcinoma reported a rather low GNAS1 mutation rate of $2.3 \%$ and also a coincidence with KRAS mutations. In addition, a correlation with right-sided location was reported, however, this finding does not correlate with our case which consisted of the recurrence of a rectal adenocarcinoma [28]. In addition, it may be interesting to compare the mutation status with the immunohistochemical expression pattern of colon tumors, e.g. markers like CDX2, desmocollin 1-3, PITXI [29] or the MSS/MSI phenotype.

Similarly to other studies [16] we did not find GNAS1 mutations in hyperplastic gastric polyps. Thus, GNAS1 mutation seems to be a rare molecular event in hyperplastic polyps. The analysis was inspired by recent studies showing GNAS1 mutations in pyloric gland adenomas [30,31]. Our results suggest that distinct genes and pathways are affected in different gastric tumor entities.

GEP-NETs are neuroendocrine tumors with a rising incidence and also represent the most common NETs [32]. Plöckinger et al. suggested surgery as the only therapeutic option for patients with GEP-NETs [33] and despite severe pharmacologic tests it is still the only option currently [34], although radionuclide therapy showed a certain constricted value [34]. Patients with GEP-NETs have a very heterogeneous prognosis, ranging from an overall five-year-survival from $100 \%$ to lower than $10 \%$ depending on the location of tumor and its biological aggressiveness [35]. Therefore, it is particularly important to identify biomarkers and better understand biology of these tumors with regard to early diagnosis and treatment. Since we did not find any GNAS1 mutation in 31 GEP-NETs, it may be assumed that this mutation is rarely involved in the development of this kind of tumors. Our findings contrast with reports which showed a detectable frequency of GNAS1 mutation in other neuroendocrine tumors, for example $40 \% \mathrm{GH}$-secreting pituitary tumors presented GNAS1 mutations [36] and toxic thyroid adenomas - 4.71\% GNAS1 mutations [37]. The mutational spectrum of GNAS1 seems also to be different in patients with pseudohypoparathyroidism [38].

In summary, GNAS1 exon 8 mutations seem to be rare events in gastrointestinal tumors. We confirmed that GNAS1 mutations may occur in tubular-villous adenoma and villous adenoma. GNAS1 mutations may be particularly important for epithelial colon tumors with villous differentiation, however, they occur very rarely in colorectal adenocarcinoma. Moreover, GEP-NETs and hyperplastic polyps of the stomach seem not to be connected to genetic mutation of GNAS1 exon 8.

\section{Acknowledgements}

The technical assistance of Kristin Zöller and Carola König is gratefully acknowledged.

\section{References}

1. Ferlay J, Steliarova-Foucher E, Lortet-Tieulent J et al. Cancer incidence and mortality patterns in Europe: estimates for 40 countries in 2012. Eur J Cancer. 2013;49:1374-1403.

2. Okines A, Verheij M, Allum W, Cunningham D, Cervantes A and ESMO Guidelines Working Group. Gastric Cancer: ESMO clinical practice guidelines for diagnosis, treatment and follow-up. Ann Oncol. 2010;21(Suppl 5):v50-v54.

3. Weinstein LS, Chen M, Liu J. Gs(Alpha) mutations and imprinting defects in human disease. Ann NY Acad Sci. 2002;968:173-197.

4. Weinstein LS, Liu J, Sakamoto A, Xie T, Chen M. Minireview: GNAS: normal and abnormal functions. Endocrinology. 2004;145:5459-5464.

5. Linglart A, Maupetit-Méhouas S, Silve C. GNAS-related loss-of-function disorders and the role of imprinting. Horm Res Paediatr. 2013;29:119-129.

6. Nagai Y, Nishimura A, Tago K, Mizuno N, Itoh H. Ric-8b stabilizes the alpha subunit of stimulatory $\mathrm{G}$ protein by inhibiting its ubiquitination.J Biol Chem. 2010;285:11 114-11 120.

7. Bastepe M. The GNAS locus: quintessential complex gene encoding Gsalpha, Xlalphas, and other imprinted transcripts. Curr Genomics. 2007;8:398-414.

8. Nerumi S, Matsuo K, Ishii T, Tanahashi Y, Hasegawa T. Quantitative and sensitive detection of GNAS mutations causing Mccune-Albright syndrome with next generation sequencing. PLoS One. 2013;8:e60525.

9. Shi RR, Li XF, Zhang R, Chen Y, Li TJ. GNAS mutational analysis in differentiating fibrous dysplasia and ossifying fibroma of the jaw. Mod Pathol. 2013;26:1023-1031.

10. Izzi B, de Zegher F, Francois I et al. No evidence for GNAS copy number variants in patients with features of Albright's hereditary osteodystrophy and abnormal platelet Gs activity. J Hum Genet. 2012;57:277-279.

11. Alves C, Sampaio S, Barbieri AM, Mantovani G. Pseudohypoparathyroidism type Ia: A novel GNAS mutation in a Brazilian boy presenting with an early primary hypothyroidism.J Pediatr Endocrinol Metab. 2013;26:557-560.

12. Mantovani G, Lania AG, Spada A. GNAS imprinting and pituitary tumors. Mol Cell Endocrinol. 2010;326:15-18.

13. Furukawa T, Kuboki Y, Tanji E et al. Whole-exome sequencing uncovers frequent GNAS mutations in intraductal papillary mucinous neoplasms of the pancreas. Sci Rep. 2011;1:161.

14. Kanda M, Knight S, Topazian M et al. Mutant GNAS detected in duodenal collections of secretin-stimulated pancreatic juice indicates the presence or emergence of pancreatic cysts. Gut. 2013;62:1024-1033.

15. Nishikawa $G$, Semine $S$, Ogawa $R$ et al. Frequent GNAS mutations in low-grade appendiceal mucinous neoplasms. Br J Cancer. 2013;108:951-958.

16. Yamada $M$, Semine S, Ogawa $R$ et al. Frequent activating GNAS mutations in villous adenoma of the colorectum. J Pathol. 2012;228:113-118.

17. Idziaszczyk S, Wilson $\mathrm{CH}$, Smith CG, Adams DJ, Cheadle JP. Analysis of the frequency of GNAS codon 201 mutations in advanced colorectal cancer. Cancer Genet Cytogenet. 2010; 202:67-69. 
18. Knösel T, Chen Y, Altendorf-Hofmann A et al. High KIT and PDGFRA are associated with shorter patients survival in gastroenteropancreatic neuroendocrine tumors, but mutations are a rare event. J Cancer Res Clin Oncol. 2012;138:397-403.

19. Bosman FT, Carneiro F, Hruban RH, Theise ND. World Health Organization, and International Agency for Research on Cancer. WHO Classification of Tumours of the Digestive System, 4th ed. IARC Press, Lyon 2010.

20. Capella C, Heitz PU, Höfler H, Solcia E, Klöppel G. Revised classification of neuroendocrine tumours of the lung, pancreas and gut. Virchows Arch. 1995;425:547-560.

21. Chung KY, Rasmussen SG, Liu T. et al. Conformational changes in the $\mathrm{G}$ protein $\mathrm{Gs}$ induced by the $\beta 2$ adrenergic receptor. Nature. 2011;477:611-615.

22. Weinstein LS, Yu S, Warner DR, Liu J. Endocrine manifestations of stimulatory $\mathrm{G}$ protein alpha-subunit mutations and the role of genomic imprinting. Endocr Rev. 2001;22:675-705.

23. Hajjhussein H, Gardner LA, Fujii N, Anderson NM, Bahouth SW. The hydrophobic amino acid cluster at the cytoplasmic end of transmembrane helix III modulates the coupling of the B1-adrenergic receptor to Gs. J Recept Signal Transduct Res. 2013;33:79-88.

24. Pierce KL, Premono RT, Lefkowitz RJ. Seven-transmembrane receptors. Nat Rev Mol Cell Biol. 2002;3:639-650.

25. Lefkowitz RJ. Seven transmembrane receptors: something old, something new. Acta Physiol (Oxf). 2007;190:9-19.

26. Tabareau-Delalande F, Collin C, Gomez-Brouchet A et al. Diagnostic value of investigating GNAS mutations in fibro-osseous lesions: a retrospective study of 91 cases of fibrous dysplasia and 40 other fibro-osseous lesions. Mod Pathol. 2013;26:911-921.

27. Sekine $S$, Ogawa R, Oshiro T et al. Frequent lack of GNAS mutations in colorectal adenocarcinoma associated with GNAS-mutated villous adenoma. Genes Chromosomes Cancer. 2014;53:366-372.

28. Fecteau RE, Lutterbaugh J, Markowitz SD, Willis J, Guda K. GNAS mutations identify a set of right-sided, RAS mutant, villous colon cancers. PLoS One. 2014;9:e87966. PubMed PMID: 24498230.

29. Knösel T, Chen Y, Hotovy S, Settmacher U, Altendorf-Hofmann A, Petersen I. Loss of desmocollin 1-3 and homeobox genes PITX1 and CDX2 are associated with tumor progression and survival in colorectal carcinoma. Int J Colorectal Dis. 2012;27:1391-1319.

30. Kushima R, Sekine S, Matsubara A, Taniguchi H, Ikegami M, Tsuda H. Gastric adenocarcinoma of the fundic gland type shares common genetic and phenotypic features with pyloric gland adenoma. Pathol Int. 2013;63:318-325.

31. Matsubara A, Sekine S, Kushima R et al. Frequent GNAS and Kras Mutations in Pyloric Gland Adenoma of the Stomach and Duodenum. J Pathol. 2013;229:579-587.

32. Faggiano A, Ferolla P, Grimaldi $F$ et al. Natural history of gastro-entero-pancreatic and thoracic neuroendocrine tumors. Data from a large prospective and retrospective Italian epidemiological study: The Net Management Study. J Endocrinol Invest. 2012;35:817-823.

33. Plöckinger $U$, Wiedenmann B. Neuroendocrine tumors of the gastro-entero-pancreatic system: the role of early diagnosis, genetic testing and preventive surgery. Dig Dis. 2002;20:49-60.

34. Baudin E, Planchard D, Scoazec JY et al. Intervention in gastro-enteropancreatic neuroendocrine tumours. Best Pract Res Clin Gastroenterol. 2012;26:855-865.

35. Gulenchyn KY, Yao X, Asa SL, Singh S, Law C. Radionuclide therapy in neuroendocrine tumours: a systematic review. Clin Oncol ( $R$ Coll Radiol). 2012;24:294-308.

36. Occhi G, Losa M, Albiger $\mathrm{N}$ et al. The glucose-dependent insulinotropic polypeptide receptor is overexpressed amongst GNAS1 mutation-negative somatotropinomas and drives growth hormone $(\mathrm{GH})$-promoter activity in $\mathrm{GH} 3$ cells. J Neuroendocrinol. 2011;23:641-649.

37. Palos-Paz F, Perez-Guerra O, Cameselle-Teijeiro J et al. and Galician Group for the Study of Toxic Multinodular Goitre. Prevalence of mutations in TSHR, GNAS, PRKAR1A and RAS genes in a large series of toxic thyroid adenomas from Galicia, an iodine-deficient area in NW Spain. Eur J Endocrinol. 2008;159:623-631.

38. Jin HY, Lee BH, Choi JH et al. Clinical characterization and identification of two novel mutations of the GNAS gene in patients with pseudohypoparathyroidism and pseudopseudohypoparathyroidism. Clin Endocrinol (Oxf). 2011;75:207-213. 